\title{
Effects of Gliclazide on Low-density Lipoprotein Oxidizability and Atherosclerosis in Cholesterol-fed Rabbits
}

\author{
Hideki Nomura, Michitaka Naito, Michiteru Kodama, Yuriko Kato, and Akihisa Iguchi \\ Department of Geriatrics, Nagoya University Graduate School of Medicine, Japan.
}

\begin{abstract}
We studied the effects of a widely-used sulfonylurea, gliclazide, on the oxidizability of low density lipoprotein (LDL) and the development of experimental atherosclerosis in cholesterol-fed rabbits. Daily oral administration of gliclazide $(20 \mathrm{mg} / \mathrm{kg} / \mathrm{day})$ tended to inhibit the aortic atherosclerosis induced by feeding a $1 \%$ cholesterol-diet for 10 weeks, although it did not affect diet-induced hyperlipidemia. The administration of gliclazide tended to inhibit the increase of serum thiobarbituric acid-reacting substances (TBARS) by cholesterol feeding and to increase the lag time of the conjugated-diene formation of LDL subjected to in vitro oxidation by copper ion, although without significance. The present study suggests that gliclazide may have antioxidative properties in vivo, and have further beneficial effects for the treatment of diabetes mellitus by inhibiting the oxidation of LDL. $J$ Atheroscler
\end{abstract} Thromb, $2000 ; 7: 104-109$.

Key words : Gliclazide, Low-density lipoprotein, Antioxidant, Atherosclerosis

\section{Introduction}

Coronary artery disease is the leading cause of death in diabetes mellitus, and diabetes confers a two- to four-fold relative risk of developing coronary artery disease (1). Even when adjusted for other risk factors such as hypertension and hyperlipidemia, diabetes remains an important cardiovascular risk factor. Diabetes causes a thrombotic tendency in patients, with increased platelet reactivity, impaired fibrinolytic activity, and increased oxidative stress (2).

It has been shown that hyperglycemia causes the production of free radicals in several ways (3). This may contribute to the development of diabetic macrovascular complications through increased oxidation of low-density lipoprotein (LDL), which is thought to be a crucial step in

Address for Correspondence: Michitaka Naito, MD, PhD., Department of Geriatrics, Nagoya University Graduate School of Medicine, 65 Tsuruma-cho, Showa-ku, Nagoya 466-8550, Japan.

Tel: $+81-52-744-2364$ (Direct dialling),

Fax: $+81-52-744-2371$

E-mail : naito@med.nagoya-u.ac.jp

Received February 1, 2000.

Accepted for publication March 20, 2000. the development of atherosclerosis $(4,5)$.

Gliclazide, a sulfonylurea in routine clinical use in many countries including Japan, has been shown to have free radical-scavenging activity in vitro and to be an effective inhibitor of in vitro LDL oxidation and more potent on a molar basis than vitamin $\mathrm{C}(6)$. This antioxidant property of gliclazide is not shared by other sulfonylureas such as glibenclamide, tolbutamide and glipizide.

The oxidative modification of LDL is considered to be an important step in the development of atherosclerosis. After initial oxidation of the polyunsaturated fatty acid component of LDL, peroxide products attack the lysine groups of apoprotein B, altering its receptor affinity and allowing uptake by the scavenger receptor on macrophages (4). Therefore, an agent that inhibits LDL oxidation may have the potential to prevent or retard the development of atherosclerosis.

In 1978, Marquie reported that the administration of gliclazide strongly inhibited the development of aortic and particularly coronary lesions induced by the atherogenic diet but did not inhibit the development of plasma lipid disturbances induced by the diet (7). However, he did not study the oxidative stress and did not discuss the antiatherogenic mechanisms of gliclazide.

In the present study, we studied the effects of gliclazide 
on LDL oxidizability and atherosclerosis induced by cholesterol feeding in rabbits.

\section{Materials and Methods}

\section{Animals and study design}

Sixteen male New Zealand White rabbits (Kitayama, Japan) were housed individually at $24 \pm 1^{\circ} \mathrm{C}$ with a 12 -hour light: dark cycle. They were allowed free access to water and commercial rabbit nonpurified diet for 10 days to enable them to adapt to the new environment. Then rabbits were divided into two groups with matched body weights and serum cholesterol and triglyceride concentrations. The control group $(n=8)$ was given a standard diet containing $1 \%$ cholesterol. The gliclazide group $(n=8)$ was given a standard diet containing $1 \%$ cholesterol and supplemented with gliclazide $(20 \mathrm{mg} / \mathrm{kg} /$ day $)$. Each $\mathrm{rab}-$ bit was given $100 \mathrm{~g}$ of its respective diet daily in the morning for 10 weeks. One rabbit in the gliclazide group died of pneumonia during the study period and was excluded from the statistical analysis. Dietary consumption did not differ between the two groups of animals during the study period (data not shown). All the protocols for animal experiments were approved by the Laboratory Animal Care Advisory Committee of Nagoya University.

\section{Blood sampling}

Blood samples after overnight fasting for 16 hours were obtained from an ear artery at the beginning of the study, after 4 weeks, and at the end of the study period (10 weeks).

After 3 weeks of treatment, blood samples were taken from 3 rabbits in each group before, 1 hour, 3 hours, and 6 hours after receiving the diet in the morning and serum glucose was measured.

Serum was stored at $-80^{\circ} \mathrm{C}$ until assay.

\section{LDL isolation and oxidation}

LDL was isolated by ultracentrifugation followed by in vitro copper-induced LDL oxidation $(8,9)$. In brief, after isolation, the LDL was dialyzed for 24 hours in the dark at $4^{\circ} \mathrm{C}$ against phosphate-buffered saline (PBS) containing $10 \mu \mathrm{mol} / \mathrm{L}$ EDTA. The LDL-containing sample was filtered through a $0.45-\mu \mathrm{m}$ filter and diluted with dialysis buffer to a final concentration of $100 \mu \mathrm{g}$ protein $/ \mathrm{mL}$. The oxidation was initiated by the addition of freshly prepared $10 \mu \mathrm{mol} / \mathrm{L} \mathrm{CuSO}_{4}$ solution. The kinetics of the oxidation of LDL were determined by monitoring the change of the 234-nm diene absorption on a UV spectrophotometer (Shimadzu, Tokyo, Japan) at $37^{\circ} \mathrm{C}$. The change of absorbance at $234 \mathrm{~nm}$ versus time was divided into three phases, ie, a lag phase, a propagation phase, and a decomposition phase. Lag time was calculated as described elsewhere.

\section{Extent of aortic atherosclerosis}

At the end of the study period, rabbits were killed by administration of a bolus injection of sodium pentobarbital. Immediately thereafter, aortas were removed from the arch to the descending thoracic aorta. The aortas were cleaned of excess adventitial tissue, and rinsed with saline and opened longitudinally. After photographing, the aortas were fixed with formalin for histological analysis. The weight of the excised heart, kidneys, and liver was also measured. The area covered by atherosclerotic lesion was quantified with a planimetry system.

\section{Other methods}

Serum total cholesterol, HDL-cholesterol and triglycerides were determined by commercially available enzymatic assay kits. Serum glucose was determined enzymatically using a glucose autoanalyzer (YSI2300 Stat Plus, Yellow Springs Instrument, Yellow Springs, $\mathrm{OH}$, USA). Protein was determined with a bicinchonic acid protein assay kit (Pierce, Rockford, IL, USA), using bovine serum albumin as the standard. The extent of lipid peroxidation in serum was determined as 2-thiobarbituric acid (TBA) reacting substances (TBARS) as described previously (10). The concentration of gliclazide in serum and LDL was measured using the HPLC method as described previously (11).

\section{Statistics}

Results are expressed as mean \pm S.D. unless otherwise stated. Statistical evaluation was performed using the paired Student's $t$ test, with a $p$ value of $<0.05$ considered to be significant.

\section{Results}

\section{Food intake and growth}

Dietary consumption did not differ between the two groups of animals during the study period (data not shown). Weight gain also did not differ between the two

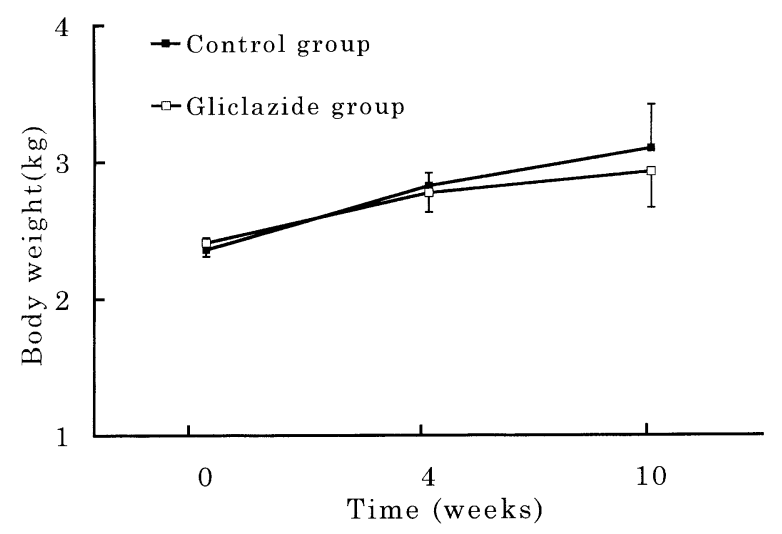

Fig. 1. Changes in body weight. Body weight did not differ between the two groups. 
A

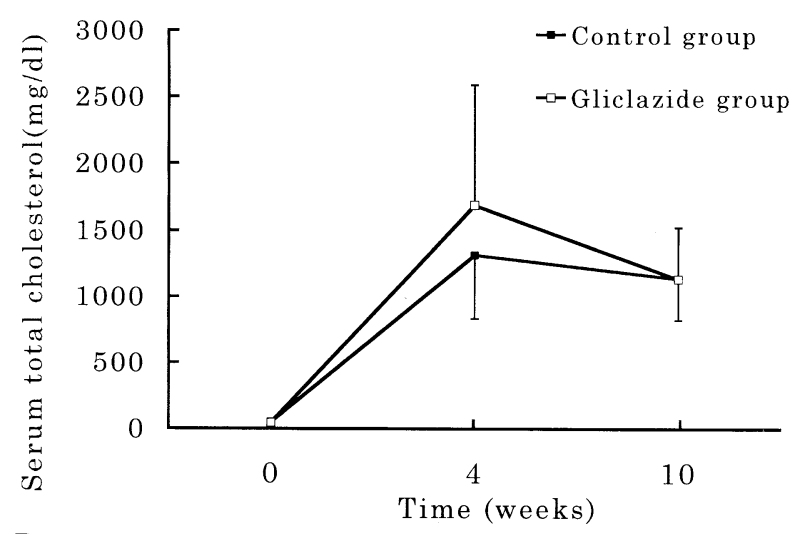

$\mathrm{B}$

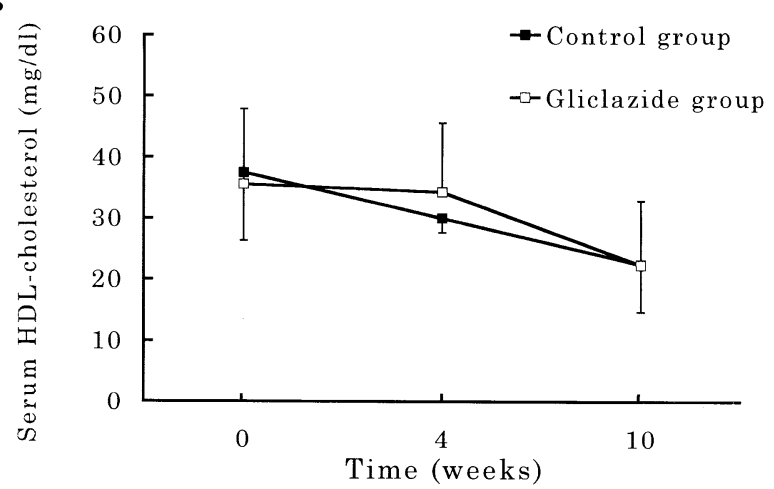

C

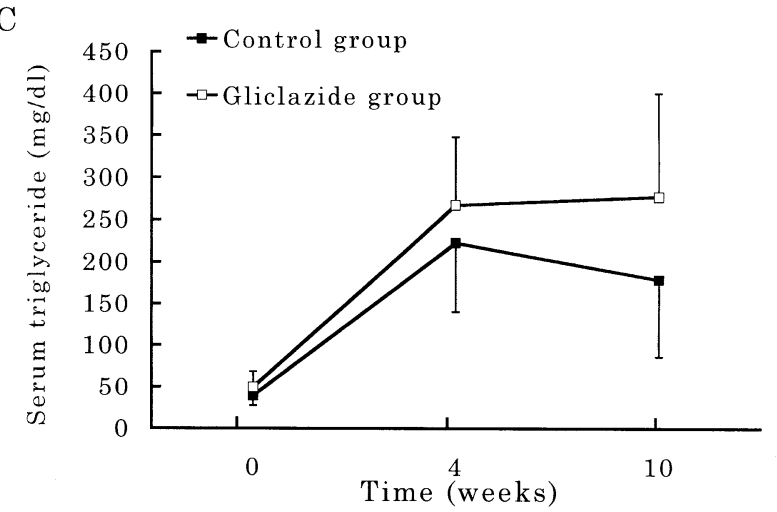

$\mathrm{D}$

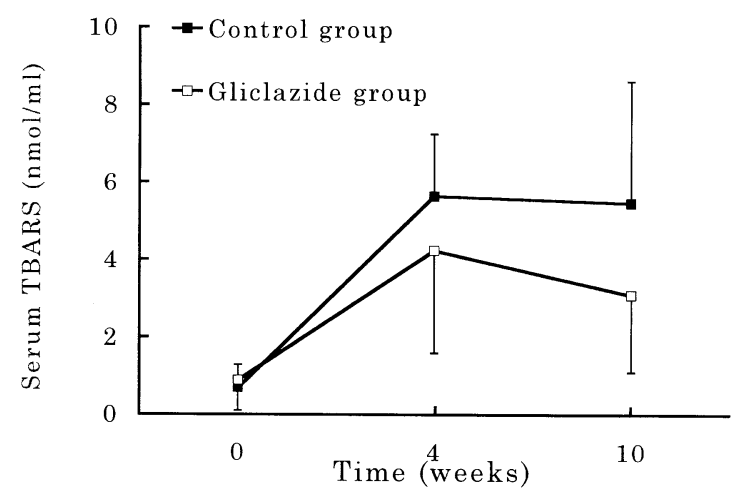

Fig. 2. Changes in serum lipids and TBARS. Serum total cholesterol (A), HDL-cholesterol (B) and triglyceride (C) levels were similar between the two groups during the study period. Serum TBARS increased with cholesterol feeding, and was slightly, but not significantly lower in the gliclazide group than the control ( $p=0.13$ at 10 weeks) (D).

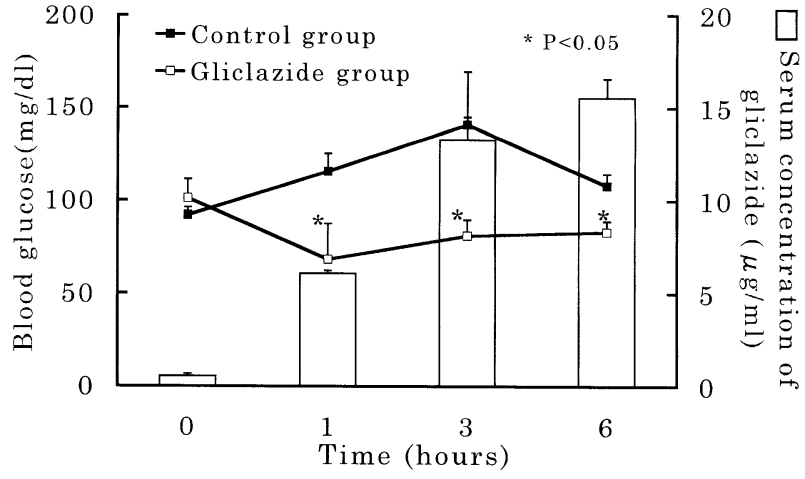

Fig. 3. Changes in blood glucose. Blood samples were taken from 3 rabbits in each group before, 1 hour, 3 hours and 6 hours after given the diet in the morning after 3 weeks and blood glucose was measured. The fasting blood glucose level was not different between the two groups. Slight decrease of blood glucose in the gliclazide group was demonstrated after receiving the diet. Serum concentrations of gliclazide in the gliclazide group are shown as the open bars. No gliclazide was detected in serum at the beginning of the experiment in the gliclazide group and in the control group during the study. groups (Fig. 1).

\section{Serum lipids and glucose}

Serum total cholesterol, HDL-cholesterol and triglyceride levels were similar between the two groups during the study period (Figs. 2A-C).

The concentration of TBARS in serum was determined as an indication of lipid peroxidation in vivo. Serum TBARS increased with cholesterol feeding, and was slightly, but not significantly, lower in the gliclazide group than in the control (Fig. 2D).

The fasting serum glucose level was not different between the two groups (Fig. 3). After 3 weeks of treatment, blood samples were taken from the 3 rabbits of each group before, 1 hour, 3 hours and 6 hours after receiving the diet in the morning. Slight increases of serum glucose were demonstrated 1 and 3 hours after feeding in the control group without significance. Slight but not significant decrease of serum glucose was demonstrated 1 to 6 hours after feeding in the gliclazide group. 


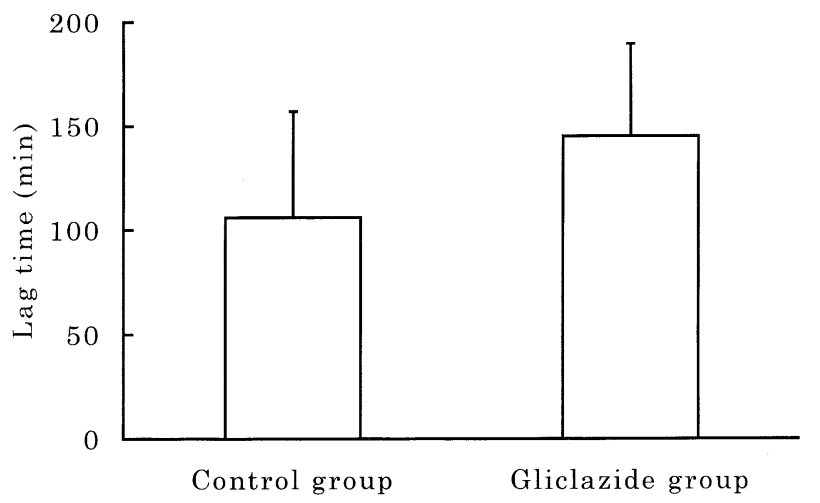

Fig. 4. Susceptibility to in vitro oxidation of LDL from rabbits fed cholesterol-rich diet with or without the supplementation of gliclazide. The oxidizability of LDL was determined in vitro by continuously measuring the conjugated-diene production induced by incubation with copper. Supplementing the rabbit diet with gliclazide resulted in a $36.7 \%$ increase in lag time after 4 weeks of treatment although without significance.

\section{Concentration of gliclazide}

The concentration of gliclazide in the serum was measured using the HPLC method (Fig. 3). The concentration of gliclazide in serum increased up to 6 hours after receiving the gliclazide-containing diet in the morning. No gliclazide was detected in serum at the beginning of the experiment in the gliclazide group or in the control group during the study. Gliclazide was not detected in LDL even after receiving the gliclazide-containing diet (data not shown).

\section{LDL oxidizability}

The oxidizability of LDL was determined in vitro by continuously measuring the conjugated-diene production induced by incubation with copper. Supplementing the rabbit diet with gliclazide resulted in a $36.7 \%$ increase in lag time after 4 weeks of treatment although without significance (Fig. 4). Prolonged treatment did not lead to a further increase in lag time.

\section{Aortic atherosclerosis}

At the end of the study period, the rabbits were killed and their aortas were removed to quantify the extent of atherosclerotic lesion formation. Gliclazide treatment tended to result in a somewhat smaller area covered with atherosclerotic lesions compared with the control, although this was not statistically significant (Fig. 5).

\section{Other organs}

No difference in the weights of heart, liver and kidneys were observed between the gliclazide group and control group (data not shown).

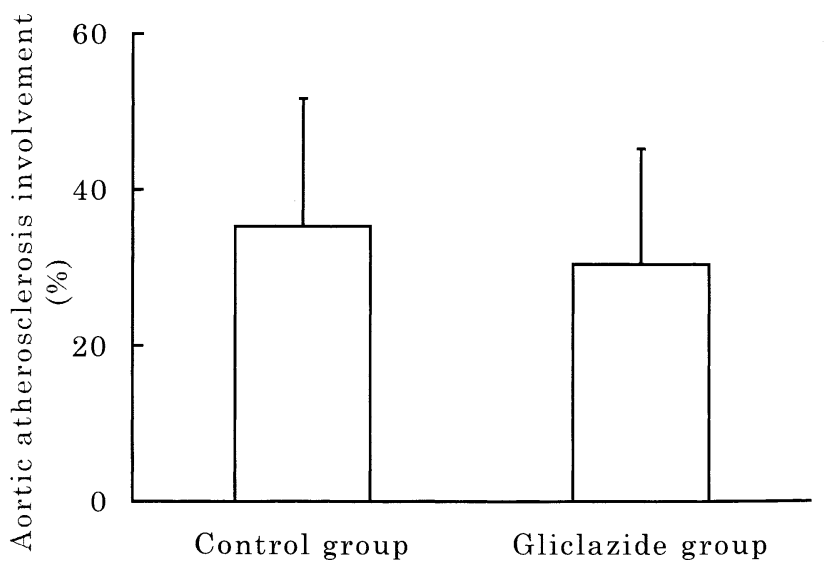

Fig. 5. Percent of aortic surface covered by atherosclerotic lesions. At the end of the study period, the rabbits were killed and their aortas were removed to quantify the extent of atherosclerotic lesion formation. $35.3 \pm 16.4 \%$ of the aortic surface of the control rabbits were covered with atherosclerotic lesions. Gliclazide treatment tended to result in a somewhat smaller area covered with atherosclerotic lesions $(29.9 \pm 16.1 \%)$, although this was not statistically significant.

\section{Discussion}

Gliclazide is a second generation sulfonylurea that is widely used in the treatment of type II diabetes mellitus and its hypoglycemic activity is well documented $(2,12)$. In addition to its metabolic effects, gliclazide has beneficial effects on the hemobiological abnormalities of type II diabetes. These effects are mediated by the azacyclooctyl ring grafted on to its sulfonylurea core (2).

Numerous studies have demonstrated that gliclazide reduces platelet hyperadhesion and platelet hyperaggregability (2). The beneficial effects of the compound on thromboxane/prostacyclin balance have been recently confirmed in type Il diabetic patients (13). Concerning fibrinolysis, gliclazide restores low plasminogen activity to normal in type II diabetic patients previously treated with first-generation sulfonylureas (14). Gliclazide increases the fibrinolytic potential by increasing endothelial cell tissue plasminogen activator and prekallikrein activity (15). More recent studies suggest that gliclazide may have effects on the fibrin network structure, rendering the fibrin more amenable to fibrinolysis (16). Diabetes was associated with significant impairment of acetylcholine-induced endothelium-dependent relaxation of the abdominal aorta which was not significant in diabetic rabbits treated with gliclazide in vivo (17). Aortas from diabetic rabbits studied in the presence of a nitric oxide synthase inhibitor, L-NAME showed an exaggerated contraction to acetylcholine which was prevented in rabbits treated with gliclazide.

Furthermore, gliclazide has been shown to have a potent free-radical-scavenging activity in vitro. Glicla- 
zide scavenges free radicals such as superoxide anion, hydroxyl radical, and nitric oxide in a dose-dependent manner $(18,19)$. The resistance to oxidation, expressed as the lag time between the addition of copper and commencement of oxidation, was significantly increased by the addtion of gliclazide in vitro (20). LDL isolated from diabetic subjects supplemented with gliclazide had an increased lag time compared with untreated LDL, although other sulfonylureas including glibenclamide, glipizide, and tolbutamide had no effect on lag time. Incubation of human monocytes and bovine endothelial cells with increasing concentrations of gliclazide (2.5 to 10 $\mu \mathrm{g} / \mathrm{ml}$ ) and native LDL induced a dose-dependent inhibition of cell-mediated LDL oxidation. In addition, exposure of endothelial cells to gliclazide and native LDL induced a dose-dependent diminution of the oxidized LDL-induced monocyte adhesion to bovine endothelial cells (20).

Diabetic patients show increased levels of circulating modified lipoproteins and enhanced oxidation of plasma LDL. Increased production of malondialdehyde, a marker of lipid peroxidation, has also been found in erythrocyte membranes of diabetic patients. Circulating levels of MDA are also higher in the plasma of diabetic patients compared with control subjects (3). Gliclazide was reported to increase serum vitamin $\mathrm{E}$ and to decrease the level of lipid peroxidation markers in LDL and HDL particles with no change in the lipid profile in a randomized study with type 2 diabetes patients (21). Treatment of type II diabetics with retinopathy by gliclazide increased antioxidant status such as the levels of plasma thiols and red blood cell superoxide dismutase activity and decreased the levels of lipid peroxides and platelet aggregation (22).

Marquie reported that gliclazide at $10 \mathrm{mg} / \mathrm{kg} /$ day p.o. significantly inhibited the development of aortic and particularly coronary lesions induced by a high cholesterol diet (7). However, he did not study the oxidizability of LDL, and the data on the degree of aortic atherosclerosis was not quantitaive. Therefore, in this study, we examined the effects of gliclazide on the experimental atherosclerosis and LDL oxidizability in the cholesterolfed rabbits. In the present study, daily oral administration of gliclazide $(20 \mathrm{mg} / \mathrm{kg} /$ day $)$ tended to inhibit the aortic atherosclerosis induced by feeding a 1\% cholesterol-diet for 10 weeks, although it did not affect diet-induced hyperlipidemia. The administration of gliclazide also tended to inhibit the increase of serum TBARS by cholesterol feeding and increase the lag time of the conjugateddiene formation of LDL subjected to in vitro oxidation although without significance. The maximal serum concentration of gliclazide after oral adminstration of $40 \mathrm{mg}$ or $80 \mathrm{mg}$ in type II diabetic patients was $2.6 \mu \mathrm{g} / \mathrm{ml}$ or $4.6 \mu \mathrm{g} /$ $\mathrm{ml}$, respectively (23). The gliclazide concentration in rabbit serum in this study was not much higher than clinical dose in Japan, which range between $40 \mathrm{mg} /$ day and $160 \mathrm{mg} /$ day. Scott et al. reported that gliclazide acts as a general free radical scavenger in a dosedependent manner in an in vitro assay system(18). Although more study is necessary, the present study suggests that gliclazide may have antioxidative properties in vivo, and have further beneficial effects for the treatment of diabetes mellitus by inhibiting the oxidation of LDL.

Acknowledgments : We thank Ms Wakako Adachi for her technical assistance.

\section{References}

(1) Donahue RP and Orchard TJ: Diabetes mellitus and macrovascular complications. Diabetes Care, 15 : 11411155, 1992

(2) Ziegler $O$ and Drouin P: Hemobiological properties of gliclazide. J Diabetes Complications, 8 : 235-239, 1994

(3) Oranje WA and Wolffenbuttel HR: Lpid peroxidation and atherosclerosis in type II diabetes. J Lab Clin Med, 134 : 19-32, 1999

(4) Steinberg D: Oxidative modification of LDL and atherogenesis. Circulation, 95: 1062-1071, 1997

(5) Naito M, Hayashi T, and Iguchi A: New approaches to the prevention of atherosclerosis. Drugs, 50: 440-453, 1995

(6) O'Brien RC and Luo M: The effects of gliclazide and other sulfonylureas on low-density lipoprotein oxidation in vitro. Metabolism, 12(Suppl 1) : 22-25, 1997

(7) Marquie: Preventive effect of gliclazide on experimental atherosclerosis in rabbits. Diabetologia, 14:269-275, 1978

(8) Kang $M$, Naito $M$, Sakai $K$, Uchida $K$, and Osawa $T$ : Mode of action of sesame lignans in protecting low ${ }^{-}$ density lipoprotein against oxidative damage in vitro. Life Sci, 66: 161-171, 2000

(9) Kang M, Kawai $Y$, Naito M, and Osawa T: Dietary defatted sesame flour decreases susceptibility to oxidative stress in hypercholesterolemic rabbits. J Nutr, 129: 1885-1890, 1999

(10) Kang M, Naito M, Tsujihara N, and Osawa T : Sesamolin metabolites distribution and inhibition of lipid peroxidation in rats. J Nutr, 128: 1018-1022, 1998

(11) Noguchi H, Tomita N, Naruto S, and Nakano S: Determination of gliclazide in serum by high-performance liquid chromatography using solid-phase extraction. J Chromatogr, 583: 266-269, 1992

(12) Jennings PE : From hemobiology to vascular disease : a review of the potential of gliclazide to influence the pathogenesis of diabetic vascular disease. J Diabetes Complications, 8 : 226-230, 1994

(13) Fu ZZ, Yan T, Chen Y-J, and Sang JQ: Thromboxane/ prostacyclin balance in type II diabetes: gliclazide effects. Metabolism, 41(Suppl 1) : 33-35, 1992

(14) Gram J, Kold A, and Jespersen J: Rise of plasma t-PA fibrinolytic activity in a group of maturity onset diabetic patients shifted from a first generation (tolbutamide) to a second generation sulphonylurea (gliclazide). J Intern Med, 225 : 241-247, 1989

(15) Gram J, Jespersen J, and Kold A: Effect of oral 
antidiabetic drug on the fibrinolytic system of blood in insulin-treated diabetic patients. Metabolism, 37 : 937-43, 1988

(16) Nair CH, Maxwell L, Wilson JD, and Dhall DP : Studies on fibrin network structure in human plasma. Part II. Clinical application: diabetes and anti-diabetic drugs. Thromb Res, 67: 477-485, 1991

(17) Pagano PJ, Griswold MC, Ravel D, and Cohen RA: Vascular action of the hypoglycaemic agent gliclazide in diabetic rabbits. Diabetologia, 41: 9-15, 1998

(18) Scott NA, Jennings PE, Brown J, and Belch JJF : Gliclazide: a general free radical scavenger. Eur $\mathrm{J}$ Pharmacol, 208 : 175-177, 1991

(19) Noda Y, Mori A, and Packer L: Gliclazide scavenges hydroxyl, superoxide and nitric oxide radicals : an ESR study. Res Commun Mol Pathol Pharmacol, 96 : 115124, 1997
(20) Desfaits A-C, Serri O, and Renier G: Gliclazide decreases cell-mediated low-density lipoprotein (LDL) oxidation and reduces monocyte adhesion to endothelial cells induced by oxidatively modified LDL. Metabolism, 46: 1150-1156, 1997

(21) Tessier D, Maheux P, Khalil A, and Fulop T: Effects of gliclazide versus metformin on the clinical profile and lipid peroxidation markers in type 2 diabetes. Metabolism, 48: 897-903, 1999

(22) Jennings PE, Scott NA, Saniabadi AR, and Belch JJF : Effects of gliclazide on platelet reactivity and free radicals in type II diabetic patients: clinical assessment. Metabolism, 41(Suppl 1) : 36-39, 1992

(23) Kanatsuna $T$ et al. : Pharmacokinetics and long-term administration with gliclazide. Jpn Pharm Ther, 10: 1507-1514, 1982 (in Japanese) 